\title{
Erratum to: Fructus mume extracts alleviate cognitive impairments in 5XFAD transgenic mice
}

\author{
Jung-Cheol Park ${ }^{1}$, Jinhua Ma ${ }^{1}$, Won Kyung Jeon ${ }^{2^{*}}$ and Jung-Soo Han ${ }^{1 *}$
}

\section{Erratum}

Following publication of the original article [1] it was brought to our attention that a grant number had been missed out in the acknowledgements of this article. At current, the acknowledgements section reads as follows:

We would like to acknowledge the financial support from the R\&D Convergence Program of NST (National Research Council of Science \& Technology, G15120 and CRC-15-04-KIST) of Republic of Korea.

However, the revised version should read as:

We would like to acknowledge the financial support from the R\&D Convergence Program of NST (National Research Council of Science \& Technology, G15120 and CRC-15-04-KIST) of Republic of Korea and a grant (K15311) from the KIOM.

Received: 25 October 2016 Accepted: 25 October 2016

Published online: 31 October 2016

\section{Reference}

1. Park JC, et al. Fructus mume extracts alleviate cognitive impairments in

5XFAD transgenic mice. BMC Complement Altern Med. 2016;16:54.

\footnotetext{
* Correspondence: wkjeon@kiom.re.kr; jshan06@konkuk.ac.kr

${ }^{2}$ Herbal Medicine Research Division, Korea Institute of Oriental Medicine, Daejeon 305-811, Republic of Korea

'Department of Biological Sciences, Konkuk University, 1 Hwayang-dong, Gwangjin-gu, Seoul 143-701, Republic of Korea
} 